\title{
An appraisal of ultrasound fetal biometry in the first trimester
}

This article was published in the following Dove Press journal:

Reports in Medical Imaging

25 August 2010

Number of times this article has been viewed

\author{
Lily K Pemberton \\ Irina Burd \\ Eileen Wang \\ Division of Maternal Fetal Medicine, \\ Department of Obstetrics \\ and Gynecology, University of \\ Pennsylvania, Philadelphia, PA, USA
}

\begin{abstract}
We evaluate the current available literature on first trimester measurement references, how critically these data have been evaluated in subsequent studies, and the generalizability of the standards across different populations. We will then discuss the significance of first trimester dating for genetic screening tests and how growth in the first trimester may predict later pregnancy outcomes, which could lead to future research to modify these outcomes.
\end{abstract}

Keywords: biometry, crown-rump length, pregnancy dating

\section{Introduction}

First trimester ultrasound is widely available and commonly done. Accurate dating influences the effectiveness of screening tests and validity of growth assessment and timing of delivery throughout the rest of the pregnancy. ${ }^{1}$ Correct dating may also decrease the rate of inappropriate diagnoses of post-term and post-date gestations as well as reduce the numbers of inappropriate inductions of labor for this indication. ${ }^{2}$ Furthermore, appropriate dating of a pregnancy is also crucial when establishing gestational age (GA) at the extremes of viability. ${ }^{3}$

The sensitivity and accuracy of these scans are affected by the gestational age at which they are performed. ${ }^{2}$ Therefore, knowledge of which biometric measurements can be used and which population growth curves in the first trimester are available can allow a clinician to perform the most accurate ultrasound dating for his/her patient population.

\section{Establishment of current dating criteria}

Traditionally, regression analysis has been used to establish the relationship between GA and a biometric fetal parameter. This method uses an independent variable [GA based on last menstrual period (LMP) or, in assisted reproductive technology (ART) pregnancies, the date of fertilization] to predict the value of a dependent variable (normal fetal size at a given GA). In order for these data to be meaningful, the clinician must know the GA of the fetus with considerable accuracy. ${ }^{4}$ True accuracy cannot be determined since the actual sizes of embryos and early fetuses are not usually available for measurement to compare to ultrasound measurements, and when actual fetuses are available the most common situation is a pregnancy loss which would not represent the norm. For this reason, the populations of women that are used for these studies usually have either a certain LMP with a history of regular menses or are women who have undergone ART.
Correspondence: Irina Burd

University of Pennsylvania,

Division of Maternal Fetal Medicine, 2000 Courtyard, 3400 Spruce Street Philadelphia, PA 19107, USA

Tel +I 2156622982

Fax + I 2155735408

Email irina.burd@uphs.upenn.edu 
The work of Robinson and Fleming in 1975 and the work of Robinson in 1973 formed the basis of first trimester dating, using ultrasound. These studies looked at crown-rump length (CRL) measurements among women with certain menstrual dating using static trans-abdominal (TA) ultrasound images. The authors then used a weighted nonlinear regression model to obtain a best-fit curve. They developed a growth chart and concluded that the $95 \%$ confidence interval for predicting GA from CRL was \pm 5 days in the first trimester. ${ }^{5,6}$ Since the initial articles, these findings have been verified, and thus CRL is thought to be the most accurate measurement for determining GA in the first trimester. ${ }^{7}$

Other studies found that variability in dating, based on CRL, increased as GA increased. ${ }^{6-8}$ These findings called into question the time period over which CRL is an appropriate measurement for determining GA. In 1992, Hadlock undertook a re-evaluation of CRL measurement in the first trimester using trans-vaginal (TV) ultrasound. Using women with certain LMPs at gestations from 5-19 weeks; Hadlock used a TV ultrasound on women with pregnancies at less than eight gestational weeks, a TA ultrasound on women with pregnancies over 14 weeks, and a combination among women with pregnancies from $8-13$ weeks. In this study, CRL as small as $2 \mathrm{~mm}$ were measured. The population was predominantly white middle class women. ${ }^{7}$ Using these data Hadlock constructed a growth curve and found that the standard deviation increased as GA increased. The authors concluded that although their findings fit with the error for the previous model of Robinson and Fleming, the error from predicting GA should not be \pm 5 days, but $\pm 8 \%$ of the GA from 5-18 weeks to account for greater variation with increasing GA. ${ }^{7}$ Both TA and TV ultrasound may be utilized for measuring CRL, but at early gestations, TV allows for better visualization of the embryonic pole. ${ }^{9}$

Since the work of Robinson, Fleming, and Hadlock, many other studies have validated this original data; this remained unchanged, despite the advent of more advanced technology. ${ }^{2-4,10} \mathrm{~A}$ weakness of these studies is that they were done in a predominantly white population and did not take maternal characteristic into account. ${ }^{2,11,12}$

\section{Gestational sac versus CRL?}

As pregnancies are being dated earlier and earlier in the first trimester, more frequent scans show a gestational sac alone and no embryonic pole. The first sign of an intrauterine pregnancy on TV ultrasound is the gestational sac, seen from gestational days 28-31. At this stage the embryo is a primitive streak, not visible on ultrasound. The gestational sac increases in size by about $1 \mathrm{~mm}$ per day. ${ }^{3}$ The earliest embryonic pole can be identified at 35 days. ${ }^{13}$ At this stage the embryo is $1-2 \mathrm{~mm}$ and increases by about $1 \mathrm{~mm}$ per day. The cephalic and caudal ends cannot be differentiated and the embryonic length is typically measured in a straight line. By about six weeks, the cephalic and caudal ends can be distinguished by the presence of the hind-brain at the cephalic pole. ${ }^{3}$ At this stage the embryonic head is flexed and it is the neck-rump length that is measured. ${ }^{3}$ At about eight weeks the embryo is about $18 \mathrm{~mm}$ long the cephalic and caudal poles are easily identified, limbs can be seen, and a true CRL can be measured. ${ }^{3}$ Ultrasound technology balances the ability to magnify the image with the available resolution, such that an embryo may be only $1+\mathrm{mm}$, but the resolution upon magnification is so poor the calipers cannot be clearly placed at the poles. Since first trimester dating charts often use CRL, at early GA when the fetal pole is not yet visualized, can gestational sac be used in its place?

The use of measuring the gestational sac to estimate GA was first described by Hellman et al in $1969 .{ }^{14}$ It was also described by Robinson in 1973 and has been examined in multiple subsequent studies. ${ }^{3,5}$ One prospective cross-sectional study compared measuring gestational sac, CRL, amniotic sac, yolk sac, and biparietal diameter (BPD) in early first trimester using TV ultrasound. This study found that measurement of mean gestational sac diameter estimated GA with variability of \pm 12 days; compared to CRL, which can be easily obtained and had a variability of \pm 8.6 days. They concluded that the CRL is more accurate and is the preferred measurement. ${ }^{10}$ Goldstein found that using a regression model and measurements of CRL from a TV ultrasound in the early first trimester, that they could predict GA within \pm 3 days..$^{15}$ The general consensus is that measuring the gestational sac is less accurate than using the CRL to estimate GA. ${ }^{3}$ The mean gestational sac diameter may be used if the embryonic pole is not visualized, with the understanding that at these very early GA there will be more error if using gestational sac diameter alone. ${ }^{9}$

The CRL is generally used for embryos from $20-60 \mathrm{~mm}$ in length. ${ }^{4}$ Many authors in the field advocate for the use of CRL prior to 14 weeks and the use of other biometric measurements like BPD, head circumference (HC), abdominal circumference (AC), and femur length (FL) after 14 weeks. In principle a single linear measurement will demonstrate less variation upon repeated measures than the variation that is amplified by using multiple biometric measures. Conversely, at 12 weeks the fetus is able to flex and extend the neck, thereby altering the CRL. Some authors have argued that BPD would be more accurate after 12 weeks 
gestation for this reason. ${ }^{2}$ One study, examining the most accurate measurement for dating at 12-14 weeks gestation among in vitro fertilization pregnancies found substantial variation in CRL after 12 weeks and concluded that this was likely largely due to fetal neck flexion and extension. ${ }^{16}$ It is recommended that the CRL measurement be performed when the fetal head is in a "neutral" position. The authors concluded that the variation in measurement of CRL may be due to having no standard definition for what a "neutral" position of the fetal head is, thereby creating variation in measurements among different examiners who were using different interpretations of the "neutral" position of the fetal head. ${ }^{16}$ Many of these other measurements are possible in the first trimester and some authors have argued that they may provide more accurate dating than CRL. These measurements are feasible at 11-16 weeks, but their accuracy in predicting the day of delivery is not clear due to the lack of reliable growth charts. ${ }^{17}$ Some authors argue that there is a necessity for growth charts for BPD, FL, and AC in the first trimester because these measurements could provide more accuracy at predicting poor first trimester growth than merely CRL. ${ }^{18}$ Authors have gone so far as to create growth charts using data they have collected. ${ }^{18}$ However, many others argue that these biometric measurements are technically difficult and difficult to reproduce among different ultrasonographers.

One study looked at pregnancies between eight and 16 weeks and measured CRL, BPD, and FL and then assessed which measurements were best for predicting the day of delivery. The study included women with both certain and uncertain LMPs. The majority of the study participants had a certain LMP. The investigators used linear regression models and compared results using different measurements. They found that CRL was more accurate than BPD at 15-60 mm, but that BPD was superior at 13 weeks and after. FL was not as accurate as either CRL or BPD. ${ }^{17}$ In second trimester fetal biometry, there is significant improvement in the accuracy of predicting GA when more than one biometric parameter is used. ${ }^{4}$ However, in this study of first trimester biometry, the authors found that combining measurements did not increase accuracy or precision, likely because since there is inherent variation with each parameter and the composite variation is then amplified when determining the overall GA. They concluded that CRL was the best measurement for up to 12.5 weeks and BPD was the best measurement after 13 weeks. ${ }^{17}$

Another study looked at the reproducibility of different fetal measurements in the first trimester and found that CRL had the highest intra- and inter-observer reproducibility. ${ }^{19} \mathrm{CRL}$ has smaller standard error than other biometric measurements and studies have shown that limb length in the first trimester is more highly correlated to CRL than to GA..$^{20,21}$ Most data indicate that CRL is still the most accurate biometric parameter in the first trimester and that fetal parts should not be measured until the 12th to 13th week of gestation.

The CRL becomes less accurate after 14 weeks GA and at that point generally $\mathrm{BPD}, \mathrm{HC}, \mathrm{AC}$, and $\mathrm{FL}$ are more accurate. In the 1991 study, ${ }^{7}$ Hadlock also concluded that CRL could accurately approximate GA in the early second trimester, but not more so than the more traditional measurements of BPD and FL. CRL is a less reliable measurement after 14 weeks and BPD and FL are generally more accurate at this GA. For women who present at or beyond 14 weeks GA, HC, or BPD is recommended over CRL. ${ }^{3}$ Ultimately it is the time frame of 12-14 weeks that may be harder to apply these recommendations, where the limitations of CRL and BPD, HC, FL, AC are apparent, particularly inopportune as many women present at this time for first trimester genetic screening (see below). A large study investigating the use of CRL together with BPD at this time frame is merited.

\section{First trimester measurement and ethnicity}

Several studies have been conducted looking at maternal factors and fetal factors and how they affect first trimester growth and growth norms. Some authors argue that there is no evidence that fetal gender and ethnicity significantly influence second trimester measurements and therefore it may be intuited that they do not affect first trimester measurements either. ${ }^{2}$ Others state that ethnicity has a significant influence on the fetal $\mathrm{BPD}, \mathrm{FL}$, and AC. They argue that the published CRL dating formulae were based predominantly on Caucasian populations and it is not known if ethnic differences in CRL exist ${ }^{12}$ or if they do exist, whether they are of clinical significance.

Bottomley et al looked at maternal age and ethnicity, and found that the rate of growth for fetuses was increased with increased maternal age and a higher growth rate among women with Black ethnic origin compared to either White or Asian maternal ethnic origin. ${ }^{11}$ Conversely, others examined the accuracy of growth charts developed by Robinson in 1973 and Hadlock in 1991.,11 Both studies found that the charts developed by Robinson and Hadlock have high correlation with CRL measurements in different populations, including Australian and ethnic Chinese populations. ${ }^{2,12}$

In reviewing the literature spanning the 1970 s to the 2000s, remarkably the dating curves from Robinson and Fleming as well as Hadlock are accurate across populations and over time. Separate dating curves for different populations do not appear necessary. 


\section{The significance of first trimester growth}

Before the widespread use of first trimester ultrasound, it was generally accepted that embryonic/fetal growth was constant in the first trimester. It was believed that first trimester growth was largely determined by genetics and variation in fetal growth rates was only seen in the second and third trimesters. ${ }^{22}$ However, more recent studies have indicated that first trimester growth varies among fetuses and may be a marker for adverse pregnancy outcomes and poor growth in the second and third trimesters. For instance, chromosomal abnormalities like trisomy 18 and triploids are associated with slow first trimester growth. ${ }^{23,24} \mathrm{~A}$ visible amniotic sac but a fetal size less than expected at seven weeks is suggestive of impending pregnancy failure. ${ }^{3}$ Several studies have shown that smaller than expected CRL is associated with increased likelihood of miscarriage. ${ }^{25-27}$ First trimester growth restriction may also be linked to adverse outcomes such as low birth weight and preterm delivery. ${ }^{3,22}$ An excellent example of this is the relationship between twin embryos in the first trimester and the later development of growth discrepancies. ${ }^{28}$

A large multicenter trial assessing the performance of first and second trimester screening for Down syndrome in the United States (FASTER trial) ${ }^{29}$ demonstrated a direct relationship between first trimester size and fetal growth in the third trimester. While the use of women who underwent ART assistance could be a strength of the study, due to issues of correct GA, this population clearly did not conceive spontaneously. Therefore this population may not be representative of the general population and may not accurately reflect normal growth. However, underscoring a true relationship between the first trimester growth and third trimester outcome is evidence that placental function in early pregnancy has a relationship to birth weight and fetuses that are smaller than expected in the first trimester are at increased risk for low birth weight. ${ }^{30,31}$ The authors concluded that since an association between first trimester growth and birth weight at or close to term in both spontaneously conceived pregnancies and in pregnancies conceived through ART were similar, the same finding would be unlikely to result from factors other than a common underlying mechanism. ${ }^{22}$ The results of this and many other studies indicate that early first trimester growth is not constant and likely affected by a myriad of maternal and fetal influences and manifests more obviously in later pregnancy outcomes. Therefore, adverse outcomes may be predicted in early pregnancy by assessment of growth in the first trimester. ${ }^{3}$

Furthermore, accurate dating is critical for an appropriate genetic screening, diagnosis, and subsequent management.
First trimester genetic screening, either alone or in sequential or integrated screening with second trimester analytes, relies on accurate dating and any inaccuracy would reduce its sensitivity, specificity, and its utility. ${ }^{32,33}$ Therefore, with the development of first trimester aneuploidy screening, the importance of reliable dating of the pregnancy has become even more paramount. ${ }^{12}$ The use of the correct first trimester dating is crucial for further management decisions, including the invasive testing such as chorionic villus sampling and amniocentesis.

\section{Conclusion}

Accurate dating in the first trimester is becoming increasingly important. Many studies have been conduced examining the best biometric measurements to complete for accurate dating in the first trimester. Amazingly, over 30 years after the original research of Robinson and Fleming and over 10 years since the work of Hadlock, the CRL tables based on this original data have been validated repeatedly over different populations of women. This leads us to conclude that CRL is still the most accurate fetal measurement in the first trimester and that these dating curves are applicable across populations.

New research is being conducted looking at using threedimensional (3D) ultrasound and using fetal and gestational sac volumes rather than 2D measurements. This body of data is still forming and deserves review as well. However, 3D ultrasonography is not universally available. Gestational sac diameter is an option in very early pregnancies if no embryonic pole is visualized; however CRL is more accurate and is the preferred method. Monitoring gestational sac size in relation to the CRL may have more utility in predicting adverse pregnancy outcomes, but should not be incorporated into protocols formula at this time if CRL data are available.

The best evidence currently is that CRL should be used in the first trimester. The ideal time to date a pregnancy in the first trimester is when there is an embryonic pole to measure and before the CRL can vary due to fetal neck extension and flexion. Ideally, this time frame is between 8 and 12 weeks. Accurate dating in the first trimester will aid in reducing the incorrect diagnosis of post-datism and inappropriate induction of labor for this indication as well as improve counseling when faced with extreme prematurity with correct GA, optimize the sensitivity and specificity of first trimester serum and nuchal translucency screening, and allow us to more accurately assess growth in the second and third trimester, and hopefully improve care for women to allow them to have the best pregnancy outcomes possible. 


\section{Disclosure}

The authors report no conflicts of interest in this work.

\section{References}

1. Gardosi J. Dating of pregnancy: time to forget the last menstrual period. Ultrasound Obstet Gynecol. 1997;9(6):367-368.

2. McLennan AC, Schluter PJ. Construction of modern Australian first trimester ultrasound dating and growth charts. $J$ Med Imaging Radiat Oncol. 2008;52(5):471-479.

3. Bottomley C, Bourne T. Dating and growth in the first trimester. Best Pract Res Clin Obstet Gynecol. 2009;23(4):439-452.

4. Degani S. Fetal biometry: clinical, pathological, and technical considerations. Obstet Gynecol Surv. 2001;56(3):159-167.

5. Robinson HP. Sonar measurement of fetal crown-rump length as means of assessing maturity in first trimester of pregnancy. BMJ. 1973; 4(5883):28-31.

6. Robinson HP, Fleming JE. A critical evaluation of sonar "crown-rump length" measurements. Br J Obstet Gynecol. 1975;82(9):702-710.

7. Hadlock FP, Shah YP, Kanon DJ, Lindsey JV. Fetal crown-rump length: reevaluation of relation to menstrual age (5-18 weeks) with high-resolution real-time US. Radiology. 1992;182(2):501-505.

8. Drumm JE, Clinch J, MacKenzie G. The ultrasonic measurement of fetal crown-rump length as a method of assessing gestational age. Br J Obstet Gynecol. 1976;83(6):417-421.

9. Lohr PA, Reeves MF, Creinin MD. A comparison of transabdominal and transvaginal ultrasonography for determination of gestational age and clinical outcomes in women undergoing early medical abortion. Contraception. 2010;81(3):240-244.

10. Grisolia G, Milano K, Pilu G, et al. Biometry of early pregnancy with transvaginal sonography. Ultrasound Obstet Gynecol. 1993;3(6):403-411.

11. Bottomley C, Daemen A, Mukri F, et al. Assessing first trimester growth: the influence of ethnic background and maternal age. Hum Reprod. 2009;24(2):284-290.

12. Sahota DS, Leung TY, Leung TN, Chan OK, Lau TK. Fetal crown-rump length and estimation of gestational age in an ethnic Chinese population. Ultrasound Obstet Gynecol. 2009;33(2):157-160.

13. Britten S, Soenksen DM, Bustillo M, Coulam CB. Very early (24-56 days from last menstrual period) embryonic heart rate in normal pregnancies. Hum Reprod. 1994;9(12):2424-2426.

14. Hellman LM, Kobayashi M, Fillisti L, Lavenhar M, Cromb E. Growth and development of the human fetus prior to the twentieth week of gestation. Am J Obstet Gynecol. 1969;103(6):789-800.

15. Goldstein SR. Embryonic ultrasonographic measurements: crown-rump length revisited. Am J Obstet Gynecol. 1991;165(3):497-501.

16. Sladkevicius P, Saltvedt S, Almstrom H, Kublickas M, Grunewald C, Valentin L. Ultrasound dating at 12-14 weeks of gestation. A prospective cross-validation of established dating formulae in in-vitro fertilized pregnancies. Ultrasound Obstet Gynecol. 2005;26(5):504-511.

17. Taipale P, Hiilesmaa V. Predicting delivery date by ultrasound and last menstrual period in early gestation. Obstet Gynecol. 2001;97(2): 189-194.
18. von Kaisenberg CS, Fritzer E, Kuhling H, Jonat W. Fetal transabdominal biometry at 11-14 weeks of gestation. Ultrasound Obstet Gynecol. 2002;20(6):564-574.

19. Verburg BO, Mulder PG, Hofman A, Jaddoe VW, Witteman JC, Steegers EA. Intra- and interobserver reproducibility study of early fetal growth parameters. Prenat Diagn. 2008;28(4):323-331.

20. Salomon LJ, Bernard JP, Duyme M, Dorion A, Ville Y. Revisiting first-trimester fetal biometry. Ultrasound Obstet Gynecol. 2003;22(1): 63-66.

21. de Biasio P, Prefumo F, Lantieri PB, Venturini PL. Reference values for fetal limb biometry at 10-14 weeks of gestation. Ultrasound Obstet Gynecol. 2002;19(6):588-591.

22. Bukowski R, Smith GC, Malone FD, et al. Fetal growth in early pregnancy and risk of delivering low birth weight infant: prospective cohort study. BMJ. 2007;334(7598):836.

23. Bahado-Singh RO, Lynch L, Deren O, et al. First-trimester growth restriction and fetal aneuploidy: the effect of type of aneuploidy and gestational age. Am J Obstet Gynecol. 1997;176(5):976-980.

24. Schemmer G, Wapner RJ, Johnson A, Schemmer M, Norton HJ, Anderson WE. First-trimester growth patterns of aneuploid fetuses. Prenat Diagn. 1997;17(2):155-159.

25. Mantoni M, Pedersen JF. Fetal growth delay in threatened abortion: an ultrasound study. Br J Obstet Gynecol. 1982;89(7):525-527.

26. Falco P, Milano V, Pilu G, et al. Sonography of pregnancies with firsttrimester bleeding and a viable embryo: a study of prognostic indicators by logistic regression analysis. Ultrasound Obstet Gynecol. 1996;7(3): $165-169$.

27. Reljic M. The significance of crown-rump length measurement for predicting adverse pregnancy outcome of threatened abortion. Ultrasound Obstet Gynecol. 2001;17(6):510-512.

28. Kalish RB, Chasen ST, Gupta M, Sharma G, Perni SC, Chervenak FA. First trimester prediction of growth discordance in twin gestations. $\mathrm{Am}$ J Obstet Gynecol. 2003;189(3):706-709.

29. Malone FD, Canick JA, Ball RH, et al. First-trimester or secondtrimester screening, or both, for Down's syndrome. N Engl J Med. 2005; 353(19):2001-2011.

30. Smith GC, Smith MF, McNay MB, Fleming JE. First-trimester growth and the risk of low birth weight. $N$ Engl J Med. 1998;339(25): $1817-1822$.

31. Smith GC, Stenhouse EJ, Crossley JA, Aitken DA, Cameron AD, Connor JM. Early-pregnancy origins of low birth weight. Nature. 2002; 417(6892):916.

32. McGalliard C, Gaudoin M. Routine ultrasound for pregnancy termination requests increases women's choice and reduces inappropriate treatments. BJOG. 2004;111(1):79-82.

33. Wald NJ, Cuckle HS, Densem JW, Kennard A, Smith D. Maternal serum screening for Down's syndrome: the effect of routine ultrasound scan determination of gestational age and adjustment for maternal weight Br J Obstet Gynecol. 1992;99(2):144-149.
Reports in Medical Imaging

\section{Publish your work in this journal}

Reports in Medical Imaging is an international, peer-reviewed, open access journal publishing original research, reports, reviews and commentaries on all areas of medical imaging. The manuscript management system is completely online and includes a very quick and fair peer-review system, which is all easy to use.

\section{Dovepress}

Visit http://www.dovepress.com/testimonials.php to read real quotes from published authors. 